Vulnerabilidades urbanas en los países andinos

(Bolivia, Ecuador, Perú)

\title{
Experiencias de gestión de riesgos en Lima: actores y territorios urbanos
}

Expériences de gestion des risques à Lima : acteurs et territoires urbains

Risk management experiences in Lima: actors and urban territories

Alexis Sierra, Jérémy Robert, Mathieu Durand y César Abad

\section{QpenEdition}

Journals

Edición electrónica

URL: http://journals.openedition.org/bifea/2433

DOI: $10.4000 /$ bifea.2433

ISSN: 2076-5827

Editor

Institut Français d'Études Andines

Edición impresa

Fecha de publicación: 1 diciembre 2009

Paginación: 777-797

ISSN: 0303-7495

Referencia electrónica

Alexis Sierra, Jérémy Robert, Mathieu Durand y César Abad, «Experiencias de gestión de riesgos en Lima: actores y territorios urbanos », Bulletin de l'Institut français d'études andines [En línea], 38 (3) I 2009, Publicado el 01 junio 2010, consultado el 17 noviembre 2020. URL : http:// journals.openedition.org/bifea/2433; DOI : https://doi.org/10.4000/bifea.2433

Les contenus du Bulletin de l'Institut français d'études andines sont mis à disposition selon les termes de la licence Creative Commons Attribution - Pas d'Utilisation Commerciale - Pas de Modification 4.0 International. 


\title{
Experiencias de gestión de riesgos en Lima: actores y territorios urbanos
}

\author{
Alexis Sierra* \\ Jérémy Robert** \\ Mathieu Durand*** \\ César Abad ${ }^{* * *}$
}

\section{Resumen}

La gestión de riesgos en Lima se hace a través de acciones puntuales, tanto espacial como sectorialmente. Esto se explica en parte por la ausencia de gestión metropolitana de la ciudad y por la fragmentación de las políticas públicas. Por ende, las acciones de gestión de riesgos se hacen esencialmente en función de movilizaciones locales, de conflictos puntuales o de apoyos de instituciones exteriores. Este artículo propone analizar una serie de acciones realizadas según las oportunidades de financiamiento o de movilización de los actores locales. Propone un panorama no exhaustivo de experiencias de gestión de riesgos en Lima y busca identificar actores, territorios y mecanismos puestos en práctica. De este modo, nos ofrece elementos de reflexión sobre las modalidades de gestión de riesgos y sobre los desafíos de la implementación de una política de gestión del riesgo a escala de la aglomeración urbana de Lima/Callao.

Palabras clave: gestión de riesgos, fragmentación urbana, ambiente urbano, cooperación, Lima/Callao

* Université de Cergy-Pontoise-IUFM, Institut de recherche pour le Développement (IRD), UR 029, Programa Pacivur. 32 Avenue Varagnat, 93143 Bondy Cedex, Francia. E-mail: alexisierra2001@ yahoo.fr

** Institut Français d'Études Andines (IFEA, UMIFRE 17, CNRS-MAEE), Casilla 18-1217, Lima 18, Perú, Université de Savoie, CISM, laboratorio Edytem (CNRS). E-mail: jeremy.robert@univ-savoie.fr

*** Institut Français d'Études Andines (IFEA, UMIFRE 17, CNRS-MAEE), Casilla 18-1217, Lima 18, Perú, Université de Rennes 2, Centre de recherche Espace et Société (UMR ESO 6590 CNRS), E-mail:m3durand@yahoo.fr

**** Egresado de la Maestría en Geografía, Mención en Gestión y Ordenamiento Territorial, Universidad Nacional Mayor de San Marcos, Lima. Becario del IFEA en 2008. E-mail: cesarabadperez@gmail. com 


\title{
Expériences de gestion des risques à Lima : acteurs et territoires urbains
}

\section{Résumé}

La gestion des risques à Lima se fait au travers d'actions ponctuelles, spatialement et sectoriellement. Cela s'explique en partie par l'absence de gestion métropolitaine de la ville et du fait de la fragmentation des politiques publiques. Les actions de gestion des risques se font donc essentiellement en fonction de mobilisations locales, de conflits ponctuels ou d'appuis d'institutions extérieures. Cet article propose d'analyser une série d'actions réalisées au gré des opportunités de financement ou de la mobilisation des acteurs locaux. II propose un panorama non exhaustif d'expériences de gestion des risques à Lima et cherche à identifier les acteurs, les territoires et les mécanismes à l'œuvre. Il apporte ainsi des éléments de réflexion sur les modalités de gestion des risques et sur les enjeux de la mise en place d'une politique de gestion du risque à l'échelle de I'agglomération urbaine de Lima/Callao.

Mots clés : gestion des risques, fragmentation urbaine, environnement urbain, coopération, Lima/ Callao

\section{Risk management experiences in Lima: actors and urban territories}

\begin{abstract}
Risk management in Lima is made through specific actions, spatially and by sectors. This situation is partially explained by the absence of metropolitan management, as well as public policy fragmentation. Risk management actions essentially are based on local mobilizations, specific conflicts, or support from outside institutions. This article suggests analyzing a series of actions implemented according to financing opportunities or the mobilization of local actors. It proposes a non-exhaustive panorama of risk management experiences in Lima and tries to identify the actors, the territories, and the mechanisms employed. Thus it provides elements for reflection on risk management modalities and on the stakes involved in the implementation of a risk management policy in an urban agglomeration on the scale of Lima/Callao.
\end{abstract}

Key words: risk management, urban fragmentation, urban environment, cooperation, Lima/Callao

A diferencia de Quito y La Paz, Lima no tiene todavía una política pública de gestión de riesgos que abarque el conjunto de la aglomeración, ni siquiera un plan de acción metropolitano frente a una amenaza determinada. En el caso del riesgo sísmico, que todos mencionan sobremanera, las autoridades tienen dificultades para implementar una política global, que tome en cuenta todos los problemas observables en la capital peruana tales como la pobreza, la urbanización descontrolada o la ausencia de preparación ante una crisis. Las iniciativas se reducen generalmente a acciones puntuales dispersas sobre el territorio urbano. Esta constatación sigue válida para los riesgos urbanos menos estigmatizados que el riesgo sísmico, tanto por parte de las autoridades como de la población. La gestión del riesgo tiene tendencia, además, a limitarse a los riesgos «naturales», dejando de lado los riesgos antrópicos, sanitarios y ambientales, a pesar de que sus manifestaciones van creciendo en Lima/Callao. 
La ausencia de política global de gestión de riesgos se explica en parte por la debilidad institucional (especialmente en este campo), exacerbada por el tamaño y la fragmentación político-institucional de la metrópoli limeña. La aglomeración cuenta con aproximadamente 8,5 millones de habitantes (censo del INEI de 2007), se extiende sobre más de $1100 \mathrm{~km}^{2}$ y está administrada por 49 municipalidades distritales y provinciales, actuando en varias escalas1 (fig. 1). De hecho, parece más difícil construir una política pública integrada e integral en este contexto que en Quito o La Paz. Además, las diferentes municipalidades no poseen los mismos recursos técnicos y financieros, según el nivel socioeconómico de sus habitantes. Los limeños y los chalacos² tampoco viven los mismos riesgos; su representación de los peligros, de las vulnerabilidades y de aquello que debe ser prioritariamente protegido difiere en función de su situación, de su historia, de sus actividades y de su organización interna.

La ausencia de una institucionalización de la gestión de riesgo a escala de la ciudad - es decir de una administración, de una reglamentación, de objetivos y de recursos específicos que contribuyan a definir una política pública de gestión de riesgos - no significa que no exista acción de prevención o de preparación. Las hay, pero a escala de los distritos, incluso de las comunidades vecinales a través de acciones puntuales, involucrando actores institucionales como ciertas ONG. Según los lugares, estas acciones se desarrollan de manera desigual, traduciéndose tanto en acondicionamientos que transforman el espacio físico como en talleres de capacitación para la población.

Proponemos aquí una breve geografía de la gestión de riesgos en Lima, estudiando los elementos de políticas (en ausencia de una real política global) y los programas existentes en varias escalas espaciales y territoriales. Esta geografía busca identificar a los actores de estas «gestiones puntuales» del riesgo, mostrar diferentes representaciones del mismo, así como diferentes temporalidades de acción. De manera voluntaria, no retomaremos aquí las acciones públicas expuestas en los artículos de J. Robert y A. Sierra (pp. 595-621, en este volumen) y de M. Durand y P. Metzger (pp. 623-646) que muestran cómo la gestión del territorio participa en la construcción de las vulnerabilidades y en la integración de las márgenes urbanas. En cambio, abordaremos otras acciones que han sido efectuadas por las municipalidades, generalmente bajo el impulso de los habitantes. Luego veremos cuál es el interés y el límite de las acciones realizadas por las ONG y por las instituciones internacionales. Por último, notaremos cómo algunos conflictos, y algunos mecanismos de cooperación, permiten iniciar una gestión de riesgos.

$1 \mathrm{Y}$ esto sin tomar en cuenta a los gobiernos regionales de reciente creación, que no hacen sino complejizar el recorte político-administrativo de la capital. La organización político-administrativa de Lima y las diferencias entre las municipalidades provinciales y las distritales estan aclaradas en el artículo de M. Durand y P. Metzger en este volumen (pp. 623-646).

2 Habitantes de la provincia del Callao. Como la aglomeración está dividida entre las provincias del Callao y de Lima, cada una tiene su municipalidad. Además, El Callao está dividido en 6 distritos y Lima en 43. 


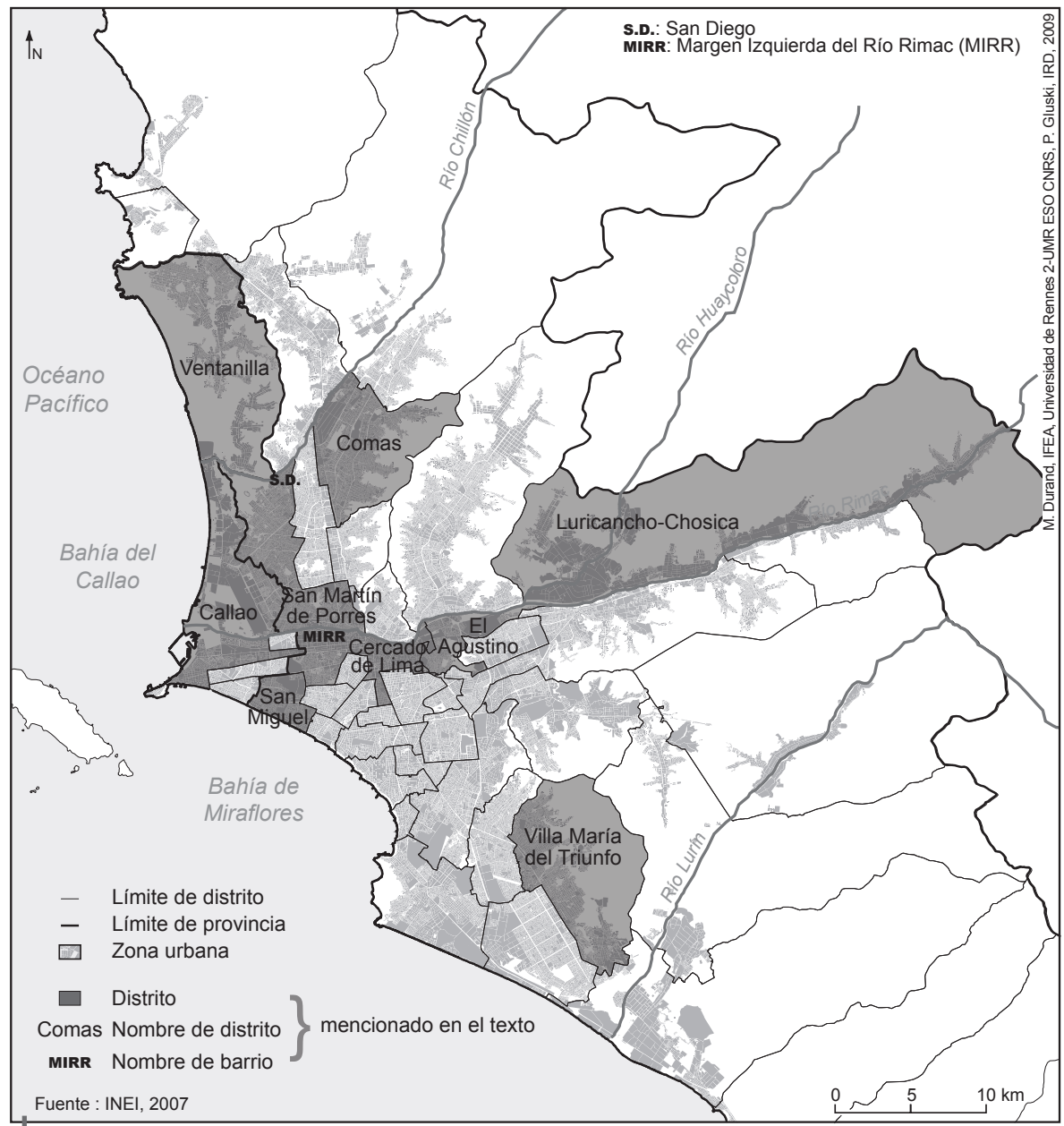

Figura 1 - Divisiones político-administrativas de Lima/Callao

\section{ACCIONES PUNTUALES DE PREVENCIÓN DE RIESGOS, REALIZADAS A PARTIR DE LA MOVILIZACIÓN DE LOS ACTORES LOCALES}

Diferentes instituciones comparten las competencias en materia de prevención de riesgos. En lo que atañe a la planificación territorial, las municipalidades provinciales reglamentan la ocupación del suelo. Disponen de planes de acondicionamiento y de desarrollo local, y tienen la obligación de tomar en cuenta los riesgos. Las municipalidades distritales realizan sus propios planes bajo la égida de las provinciales. La evaluación de los riesgos incluida en estos planes es realizada 
por los servicios de Defensa Civil3 de cada municipalidad distrital cuando esta tiene la capacidad de hacerlo, pero en la mayoría de los casos, con el apoyo de la Defensa Civil provincial. Esta se limita generalmente a los riesgos «naturales». Las modalidades de evaluación de los riesgos son definidas por el Indeci (Instituto Nacional de Defensa Civil) que ofrece un apoyo técnico: capacitación del personal, manuales de evaluación de los riesgos (Indeci, 2006), etc. La inclusión de los riesgos en los documentos municipales de planificación (provincia y distrito) es, sin embargo, bastante somera y difiere mucho de una municipalidad a otra. Además, algunas instituciones públicas realizan también estudios sectoriales de riesgos, pero generalmente de manera aislada.

En ausencia de una instancia metropolitana lo suficientemente poderosa en términos de gestión de riesgos, y de una falta de capacidad y de coordinación entre instituciones productoras de documentos técnicos, observamos el predominio de una gestión de riesgos a escala local a través de intervenciones puntuales, más que una política de lucha contra el riesgo. En efecto, por falta de gestión metropolitana, los poderes locales, a través de las 49 municipalidades de la ciudad, tienen una competencia ampliada de gestión del territorio de manera general y de los riesgos en particular. A este nivel, podemos identificar más fácilmente identificar los factores que concurren en la implementación de gestiones (frecuentemente puntuales) de los riesgos. Por ejemplo, las representaciones sociales que orientan las acciones de prevención o el ordenamiento concreto de algunas porciones del espacio urbano (las riberas de los ríos y las quebradas) consideradas como focos de amenazas. Las funciones de los diferentes actores que entran en juego serán detalladas a través de diferentes casos, así como la parte correspondiente a la movilización de la población.

\section{1. Acciones concertadas en Villa María del Triunfo}

El distrito de Villa María del Triunfo (VMT) está ubicado en la periferia sur de la aglomeración, y se ha desarrollado sobre las laderas de la Cordillera de los Andes (fig. 1). Los barrios populares, caracterizados por la precariedad de las construcciones y de las condiciones de vida, están aquí expuestos a deslizamientos de tierra, a hundimientos $y$, de hecho, a sismos. La utilización de materiales rudimentarios (madera, plásticos, etc.) y la mala calidad de las instalaciones eléctricas favorecen el riesgo de incendios. La magnitud y la dinámica de la urbanización en VMT supera las capacidades de control de la municipalidad. En ausencia de políticas definidas de lucha contra el riesgo, esta interviene de manera puntual, mayormente realizando obras de protección. Los lugares de las intervenciones son escogidos en función de las experiencias individuales acumuladas por los funcionarios de Defensa Civil y de las reivindicaciones de la población. Esta última tiene la posibilidad de convertirse en un actor válido a través del presupuesto

3 Defensa Civil es un servicio municipal encargado de la seguridad civil. Todo municipio dispone de un servicio de Defensa Civil. 
participativo que permite definir proyectos y sectores de intervención para el año venidero. Sin embargo, solamente una pequeña parte de los proyectos propuestos son efectivamente realizados por la municipalidad, incitando a algunos barrios a recurrir a un apoyo exterior: generalmente el ministerio de Vivienda, Construcción y Saneamiento o, más puntualmente, las ONG. Por su parte, la Defensa Civil distrital interviene evaluando los riesgos. Por ende, emite su propia opinión y evalúa la viabilidad técnica de los proyectos seleccionados durante el presupuesto participativo. Sin embargo, como no dispone sino de pocos recursos humanos y financieros, tampoco tiene la capacidad suficiente para asumir el conjunto de las tareas que le son encomendadas, y que van desde la evaluación de riesgos para la planificación territorial hasta la gestión de las emergencias.

Frente a las reivindicaciones de las poblaciones y a las realidades del campo, la Defensa Civil es consciente de los vacíos en la prevención de los riesgos. Por lo tanto, intenta fortalecer la preparación de las poblaciones mediante la organización de capacitaciones en primeros auxilios y a través de la difusión de información sobre los comportamientos que hay que tener en caso de accidente o de desastre (fig. 2). Frente a la urbanización informal, particularmente importante en las laderas, la Defensa Civil organiza igualmente talleres de capacitación en técnicas de construcción. Estos talleres buscan fortalecer las pircas 4 para minimizar el riesgo de hundimiento/derrumbe, pero actuan también a reducir el riesgo

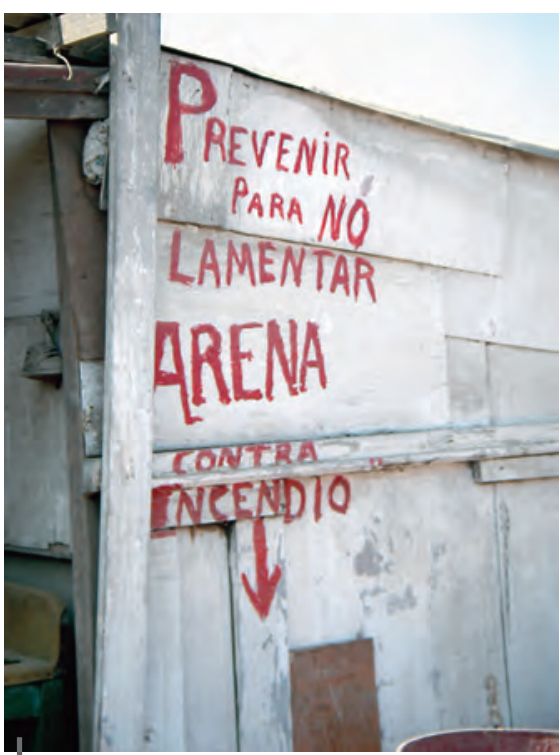

Figura 2 - Inscripciones de medidas de prevención contra incendios

Foto: J. Robert, 2009 sísmico. De este modo, unas treinta personas han sido capacitadas en el transcurso del año 2008 en el distrito. Tras esta experiencia, el objetivo actual es de realizar estas capacitaciones en los barrios considerados como más expuestos a las amenazas.

La integración del riesgo en las diferentes políticas municipales está en sus inicios, tanto en términos de vivienda y de planificación urbana como también de gestión de los residuos. Lo observamos en Villa María del Triunfo, y se puede aplicar al conjunto de los distritos de la ciudad de Lima/Callao. En vez de una política global de gestión del riesgo, existe una serie de intervenciones municipales puntuales, sobre todo acciones de sensibilización. Estas últimas contribuyen a una toma de conciencia progresiva sobre la necesidad de manejar los riesgos, pero no por ello son suficientes.

4 Micro rellenos sostenidos por muros con piedras sin argamasa o sacos de arena. 


\section{2. El acondicionamiento de las riberas del Chillón y del Rímac contra el riesgo de inundación}

En ausencia de políticas integrales de gestión de riesgos, las municipalidades implementan programas específicos que apuntan a las amenazas más visibles. Es el caso, por ejemplo, del riesgo de inundación relacionado con los diferentes ríos de la ciudad. La megalópolis limeña está atravesada por el valle del río Rímac, donde el proceso de urbanización se inició en el siglo XVI; el valle del río Lurín, al sur; y el valle del río Chillón, al norte. Estos valles han sido frecuentemente inundados en función de las crecidas iniciadas en los Andes, que permitieron su cultivo multisecular. Si las riberas del Rímac conocen una urbanización desde la época colonial, este proceso se ha generalizado y acentuado a partir de mediados del siglo XX. Las riberas de los demás ríos conocen una expansión urbana contemporánea y sus valles forman, cada uno, un eje del frente de urbanización de la ciudad.

\section{2. 1. Las riberas del río Chillón en San Martín de Porres}

Las riberas del Chillón han sido parcialmente acondicionadas con terraplenes para proteger los espacios urbanizados frente a las crecidas estacionales y a las inundaciones asociadas. Este valle presenta riesgos hasta los confines de lo urbano y de lo rural, y numerosos terrenos son aún agrícolas.

La presencia de los diques está, de hecho, estrechamente ligada a la urbanización. En algunos casos, estos son realizados para poder lotizar y urbanizar terrenos agrícolas; en otros, para proteger de las inundaciones a la urbanización ya existente. Es el caso por ejemplo de los que protegen la urbanización San Diego5, construidos primero por los habitantes y consolidados, posterior y paulatinamente, con el apoyo de las autoridades (figs. 3 y 4). Estos diques están generalmente constituidos de desechos y sobre todo de escombros. Para los recicladores, para las empresas privadas, pero también para las municipalidades y los promotores se trata de deshacerse de sus desechos preparando al mismo tiempo el terreno para la urbanización (cf. M. Durand y P. Metzger en este volumen, pp. 623-646).

Al permitir ganar tierras sobre el lecho mayor del río Chillón y lotizarlas, estos acondicionamientos muestran claramente una relación dinámica entre la gestión de riesgos y la urbanización. En el caso de la urbanización San Diego, el dique no ha sido hecho de una sola vez. La última fase de las obras se realizó tras la inundación de una parte del barrio, el 15 de marzo de 2001, consecutiva a la ruptura del dique. Según el Indeci, 388 viviendas fueron dañadas, dejando cerca de 2000 personas afectadas. Tras su erosión, el dique tuvo que ser reforzado, así como también las bases de un poste de línea de alta tensión que habían sido destruidas. La obra fue inaugurada en 2009 y realizada dentro del marco del programa nacional «Construyendo Perú». La asociación del barrio y los habitantes 


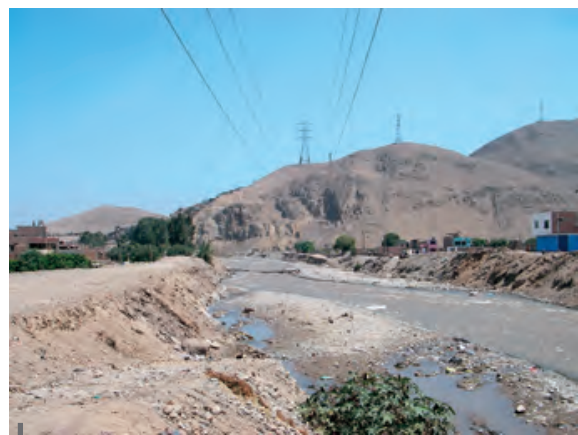

Figura 3 - El Chillón y sus riberas

Tramo de diques más consolidado sobre la ribera izquierda que sobre la ribera derecha, debido a diferencias de niveles socioeconómicos entre los barrios ubicados a orillas del río. Estos diques están hechos en gran parte con residuos y escombros

Foto: A. Sierra, 2009

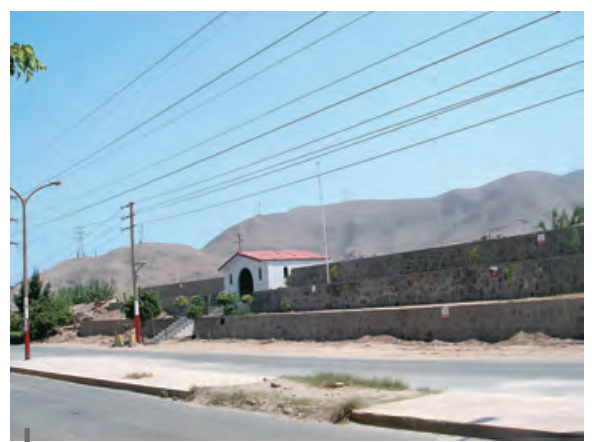

Figura 4 - Obra de protección a lo largo del río Chillón

Tramo de dique consolidado por los habitantes y las municipalidades. A pesar de ello, el agua se infiltra debajo del dique hasta las casas

Foto: A. Sierra, 2009

efectuaron las operaciones con el apoyo financiero y técnico del ministerio de Trabajo y de Promoción del Empleo (MTPE). El dique fue poco a poco arborizado e integrado al paisaje urbano.

En la parte baja del barrio existen porciones de suelo acondicionadas en jardines para las casas que están frente al dique y construidas en el lecho mayor. En este último caso, el talud del dique es a veces cavado por los residentes. Por ende, todo el proceso de urbanización parece borrar al río del paisaje. De hecho, el barrio le da la espalda al Chillón (así como a los barrios más pobres situados en la margen derecha). El dique, en tanto que obra de protección, tiende a convertirse solo en un elemento del paisaje urbano de San Diego y favorece la consolidación de la urbanización. Al mismo tiempo, modifica las dinámicas del ambiente físico y este proceso tiende a reproducirse a lo largo del río. En razón de la elevación del Chillón, los terrenos urbanizados se sitúan ya más abajo del río.

La situación de las riberas del Chillón nos recuerda a la de las riberas del río Rímac (J. Robert y A. Sierra en este volumen, pp. 595-621). En ambos casos, la urbanización se ha hecho sobre terrenos agrícolas e inundables, progresivamente rellenados con escombros y desechos. Si bien en términos de riesgos la situación no parece a priori tan dramática a lo largo del Chillón, encontramos procesos similares de construcción del riesgo: ocupación de terrenos expuestos a las amenazas, modificación de los procesos físicos generadores de riesgo y creación de amenazas (inestabilidad potencial del terreno y focos de infección posible debido a los depósitos de basura).

Estos procesos de acondicionamiento son, igualmente, un modo de lucha espontáneo contra el riesgo, común en la ciudad. De igual modo que en el río Rímac, los vecinos toman la iniciativa de obras puntuales que les atañen directamente. 
Estos acondicionamientos pueden ser financiados dentro del marco de programas sociales nacionales o municipales, sin que por ello signifiquen la concretización de un documento de planificación global, ni siquiera a escala del distrito.

\section{2. 2. Las riberas del río Rímac en El Agustino}

Un proceso similar de acondicionamiento existe a lo largo del Rímac, en particular a nivel del barrio Vicentelo Bajo en el distrito de El Agustino. Según testimonios de la población, se produjeron inundaciones en la parte alta en los años 1990. Los dirigentes del comité de residentes destacan su vulnerabilidad e insisten sobre el hecho de que el suelo urbano es producto de un relleno del lecho mayor del Rímac. Ellos se consideran expuestos a probables inundaciones y, en consecuencia, militan por una canalización del Rímac que les protegería de las crecidas. Como a lo largo del Chillón, se ha construido un dique que sostiene una línea de alta tensión. Al contrario del caso anterior, los residentes hablan del dique para enfatizar el riesgo de inundación, por lo menos en la actualidad. En efecto, la municipalidad de El Agustino tiene previsto valorizar las riberas mediante el acondicionamiento de espacios verdes y de diversión, lo que podría reducir el sentimiento de inseguridad.

Estos diques constituidos de rellenos deben supuestamente proteger el sector contra las inundaciones que tienen periodos de retorno de entre 100 y 500 años (Coopi, 2008). Sin embargo, estos contribuyen en la modificación de las condiciones de escorrentía, favoreciendo la canalización del río y creando una incertidumbre en lo que atañe a las amenazas futuras.

\section{3. Acondicionar las quebradas de Chosica contra los huaycos}

En el distrito de Lurigancho-Chosica se observa una situación similar en cuanto a la movilización de los habitantes para la reducción local de riesgos. Este distrito es afectado frecuentemente por huaycos 6 y deslizamientos de tierra. El territorio de este distrito, instalado a lo largo de la Carretera Central que sale de Lima rumbo a la Cordillera de los Andes, está marcado por numerosas obras de ingeniería civil: muros de contención sobre las laderas de los cerros, canalización parcial del Rímac, diques en el fondo de las quebradas afluentes del Rímac, etc.

Las quebradas Quirio y Pedregal están entre los sectores considerados críticos por las autoridades distritales y metropolitanas, así como por la población. A partir de los huaycos ocurridos en la década de 1980, estas quebradas han sido objeto de preocupación en torno a la gestión de los riesgos de inundaciones torrenciales, principalmente por parte de la población apoyada por algunas organizaciones no gubernamentales. La ONG Predes (Centro de Prevención de Desastres) tuvo un papel muy importante en la elaboración de estudios, asesoría técnica y capacitación en relación a la gestión de riesgos.

6 Término utilizado en el Perú para designar un flujo de lodo y escombros. 
La magnitud de los eventos y de los daños asociados facilitó la inversión, esencialmente para la realización de obras de reducción de la amenaza. Cabe subrayar que los huaycos del año 1987 provocaron el corte de la Carretera Central y dañaron el sistema de abastecimiento de agua potable (ver el artículo de C. Abad en este volumen, pp. 475-486). Entre las principales medidas de prevención se destaca la construcción de obras, principalmente diques y muros de contención. Al final de los años 1980, el Estado peruano realizó la limpieza de los cauces en ambas quebradas. Posteriormente, a mediados de los años 1990, se construyeron diques en sentido perpendicular al eje de la quebrada, con el apoyo del ministerio de Vivienda y con el asesoramiento técnico de Predes. Un total de 36 diques en la quebrada Pedregal y de 15 en la quebrada Quirio cumplen con la función de regular la pendiente, disminuyendo la velocidad de los flujos y reteniendo el material sólido transportado.

En los últimos años, los pobladores tuvieron que encontrar una manera de complementar el financiamiento. En la quebrada Quirio por ejemplo (específicamente en el barrio de Nicolás de Piérola), los pobladores consiguieron un financiamiento parcial para la construcción de diques y muros de contención por parte del programa «A Trabajar Urbano» del ministerio de Trabajo (fig. 5). El proyecto consiste en la construcción de un muro de contención a lo largo de la quebrada y de un conjunto de diques para reducir el perfil de la pendiente, transformando el fondo de la quebrada en escalera. Los diques de piedra deben retener el material acarreado por los huaycos y, al mismo tiempo, dejar pasar el agua. Al final, el perfil de la quebrada está fuertemente antropizado para reducir la fuerza del flujo y la erosión lateral. El Estado ha proveído el estudio técnico, los equipos y los materiales. La comunidad local, por su parte, proveyó la mano de obra. Este proyecto, realizado en 2007, fue financiado en $3 / 4$ por el Estado y en $1 / 4$

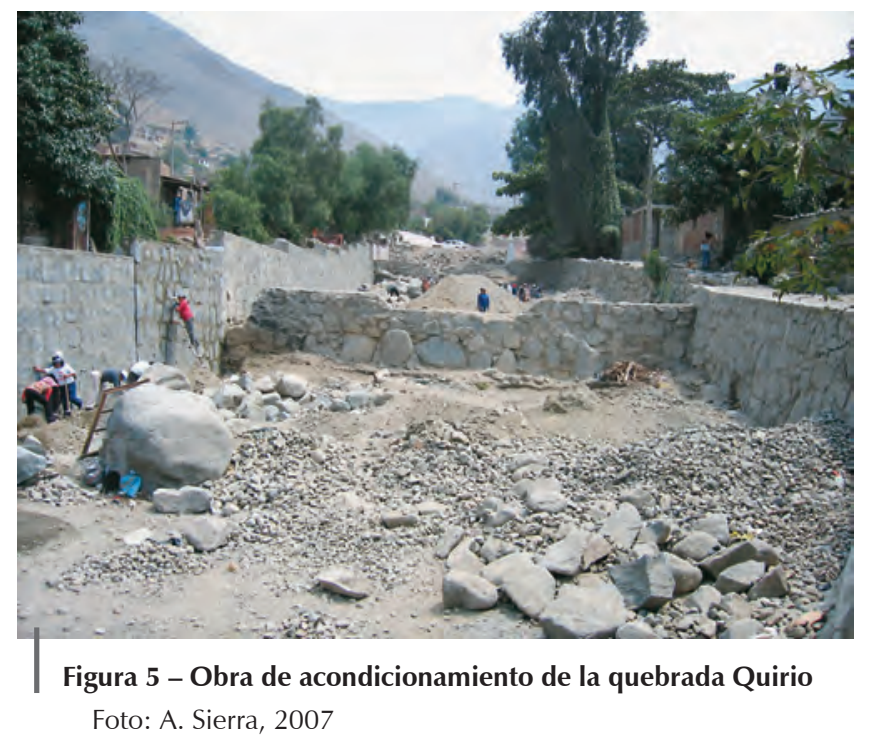


por la organización vecinal del barrio Nicolás de Piérola. Existen también casos de auto-financiamiento, como en San Miguel de Pedregal, en la parte baja de la quebrada Pedregal, donde se realizó la construcción de un muro de contención de 372 metros y la limpieza del cauce.

Esta gestión del riesgo muestra sin embargo varias debilidades. Una vez construidas las obras, los costos de mantenimiento plantean problemas. A lo largo del tiempo, la falta de mantenimiento incrementa la vulnerabilidad por la propia pérdida de funcionalidad de las obras. Luego de los huaycos de enero de 2009, muchos diques quedaron colmatados. Aunque el municipio coordinó obras de recuperación con maquinarias del Ejército, no todos los diques fueron descolmatados, provocando múltiples solicitudes a diferentes instituciones por parte de los dirigentes vecinales.

Por lo tanto, las políticas de lucha contra el riesgo se limitan a la realización de obras de lucha contra las amenazas y esta depende en gran medida del poder organizativo y reivindicativo de las comunidades. Las iniciativas de las comunidades traducen una falta de coordinación institucional y un apoyo esporádico de las autoridades públicas. Por último, no existe (o muy poco) control de la urbanización en estas zonas, a pesar de estar reconocidas como riesgosas. En el marco del programa «Ciudades Sostenibles»7, implementado por el PNUD (Programa de las Naciones Unidas para el Desarrollo), el Indeci ha realizado un estudio de amenaza con el objetivo de proporcionar elementos para la definición de la ocupación del suelo. Sin embargo, hasta el momento las recomendaciones siguen sin efecto.

El conjunto de los casos anteriores muestra la existencia de acciones concretas de lucha contra el riesgo que parecen responder a 3 características:

- las operaciones puntuales de protección contra el riesgo se originan en la iniciativa local, de los residentes o de las municipalidades de distrito con el apoyo eventual de programas nacionales;

- las acciones atañen principalmente a la amenaza para reducir su fuerza e impacto;

- los espacios de intervención dependen de desafíos específicos de desarrollo urbano.

Frente a la multiplicidad de los actores y de los territorios, y en ausencia de una institución coordinadora fuerte, no existen mecanismos que permitan integrar las intervenciones puntuales a una gestión más global del riesgo. Esta falta de coordinación se traduce más que todo en los lugares de intervención, que no siguen lógicas territoriales claras, pero parecen responder más bien a reivindicaciones o preocupaciones puntuales. Esta lógica de intervención aparece también en el marco de la cooperación internacional. En efecto, la fragmentación de la gestión del riesgo observada y la escasa capacidad de intervención de las autoridades locales, lleva a los habitantes, y a veces a las municipalidades, a buscar un apoyo

7 Este programa ha sido realizado en un centenar de ciudades a escala nacional, pero en Lima, solamente en Lurigancho-Chosica. 
técnico y financiero exterior, a través de ONG y de la cooperación internacional. Entonces las acciones están orientadas y localizadas en lugares percibidos como «riesgosos».

\section{LA GESTIÓN DE RIESGOS A TRAVÉS DE LA COOPERACIÓN INTERNACIONAL}

La relación entre los gobiernos locales y las ONG es, en muchos casos, ambigua. A pesar de que apoyan a la reducción de riesgos a nivel local, las acciones de las ONG y de las instituciones internacionales son raramente concertadas con las autoridades y tampoco están basadas en una lectura espacial de conjunto. La reducción de los riesgos se limita, tanto aquí como anteriormente, a acciones puntuales orientadas hacia el tratamiento de las condiciones sociales precarias.

Siendo una metrópoli del Sur, Lima es un campo predilecto de acción para las ONG y las instituciones internacionales de cooperación para el desarrollo. El barrio de la MIRR (Margen Izquierda del Río Rímac), aunque situado a proximidad inmediata del centro histórico de Lima, presenta todas las características de una margen urbana: espacio de relegación, de «no-derecho» y de transición (Sierra \& Tadié, 2008). Las autoridades no efectúan allí una política de desarrollo sostenida y de largo plazo. A diferencia de otros territorios de la ciudad, este barrio no tiene estrategia local que permita atraer actividades económicas. Por esta razón, esta situación de margen ha preocupado y atraído a actores del desarrollo como son las ONG. En la MIRR, dos programas consecutivos de reducción de riesgos se han desarrollado. El primero, iniciado en 2007 y de una duración de 18 meses, ha consistido en establecer un diagnóstico sobre los riesgos en la zona y capacitar a la población ante un desastre. El segundo es un programa de investigación-acción de cuatro años, iniciado en 2008, que apunta a reducir la vulnerabilidad. Comparten el apoyo financiero e institucional de organizaciones extranjeras de países del Norte: de la Comisión Europea en el primer caso y del IDRC (International Development Research Center) de Canadá en el segundo.

\section{1. Programa Dipecho de la Comisión Europea realizado por la ONG italiana Coopi}

Interesada por la reducción de la vulnerabilidad de diferentes sectores de la ciudads, la ONG italiana Coopi (Cooperazione Internazionale) inició en 2007 un proyecto financiado por el programa de preparación ante desastres de la oficina de ayuda humanitaria de la Comisión Europea (Dipecho9).

8 El proyecto abarca igualmente 6 asentamientos humanos del distrito de El Agustino.

9 Dipecho: Programa de preparación ante desastres de la Oficina de Ayuda Humanitaria de la Comisión Europea (Disaster Preparedness of the European Commission Humanitarian aid Office). 
El proyecto, intitulado «Fomentar la participación de jóvenes e instituciones locales en actividades de preparación ante desastres en dos distritos de Lima Metropolitana, Perú» (fig. 6), fue presentado en colaboración con la municipalidad de Lima y el IRD (Institut de Recherche pour le Développement). La acción se organizó en 4 ejes:

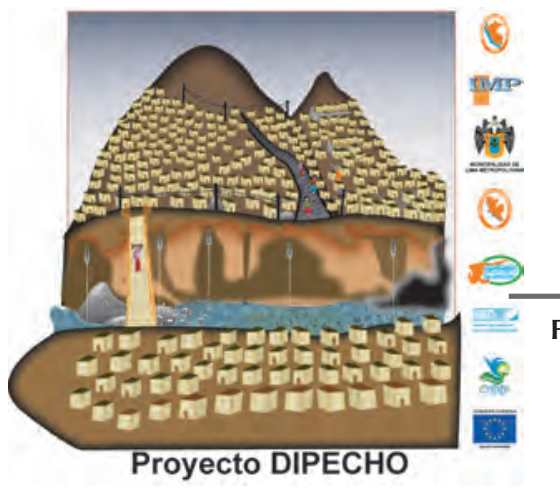

Figura 6 - Logo del proyecto Dipecho que simboliza el barrio de la MIRR, en primer plano, separado por el río Rímac de los barrios de El Agustino, en segundo plano

- la realización de estudios de riesgo en toda la MIRR, lo que incluye un estudio cartográfico (Coopi, 2008);

- la sensibilización de la población a través de talleres de información y de actividades, en particular con jóvenes;

- el fortalecimiento de las capacidades de la Defensa Civil municipal (capacitación, equipamiento, implementación de un Centro Operativo de Emergencia - COE, etc.). Las intervenciones también han apuntado a la población residente en estos barrios, a través de la capacitación de brigadistas (socorristas voluntarios), la realización de simulacros de evacuación, la instalación de una señalización que indique los lugares seguros y las rutas de evacuación previamente definidas;

- por último, se han realizado directamente algunas obras de ingeniería civil con la perspectiva de facilitar el manejo de crisis; por ejemplo, la construcción de escaleras en las zonas de difícil acceso y de un local comunitario destinado a servir de albergue.

El espacio de acción comprendía al conjunto de los barrios instalados a lo largo del Rímac. La población participó activamente en la ejecución del programa, incluso como mano de obra en las obras de reforzamiento de las estructuras. Sin embargo, se trata de un programa esencialmente social que atañe menos al ordenamiento físico del espacio que a la percepción y a los comportamientos de los habitantes. El enfoque consiste en preparar a estos barrios para una situación de crisis y aumentar la resiliencia en la perspectiva de un desastre, en particular de un sismo mayor. 


\section{2. Programa canadiense del IDRC efectuado por el IMP y la ONG peruana Cenca}

La situación de marginalidad ha motivado también la atención del IMP10 (Instituto Metropolitano de Planificación) y de la ONG peruana Cenca (Instituto de Desarrollo Urbano) para presentar conjuntamente con el IRD un proyecto de «investigación-acción participativa» ante el IDRC. El proyecto «Investigación integrada y participativa para la reducción de la vulnerabilidad, pobreza y cargas ambientales en un área crítica urbana: la Margen Izquierda del Río Rímac-Cercado de Lima» apunta a reducir la vulnerabilidad frente a los desastres de origen natural y antrópico, pero también las vulnerabilidades socioeconómicas (pobreza) y ambientales (contaminaciones diversas). Uno de los objetivos del proyecto, que privilegia una metodología de investigación participativa, es la formulación de una proposición de ordenamiento de la MIRR, basada en el diagnóstico de las diferentes vulnerabilidades de la zona. Con el objetivo de impulsar nuevas dinámicas territoriales, económicas, de organización social y de cooperación interinstitucionales, las acciones se apoyan en proyectos piloto tales como:

- la rehabilitación y el acondicionamiento de espacios públicos ocupados ilegalmente;

- el reforzamiento de la estructura de un centenar de casas;

- la reorganización de la actividad de reciclaje de los residuos sólidos, que marca fuertemente la identidad territorial del sector, y la construcción de una plataforma que concentre las actividades de los recicladores.

El desarrollo de estos proyectos piloto tiene por objetivo implementar una dinámica local, que apunte a una reducción de la vulnerabilidad. El proyecto insiste, por ello, en la capacitación y la participación local, así como en la implementación de mecanismos de coordinación de las instituciones susceptibles de intervenir en este campo.

\section{3. Dos enfoques distintos de reducción de riesgo}

Por el momento, ya que se trata de proyectos recientes, no es posible hacer una evaluación global. Pero la presentación de estos dos programas es una ocasión para analizar los diferentes enfoques de tratamiento de los espacios de riesgo: por un lado, una acción principalmente dirigida hacia la población (proyecto Dipecho), y por otro lado, una intervención que apunta al desarrollo territorial (proyecto IDRC).

La elaboración del proyecto IDRC ha sido una ocasión para poner énfasis en algunos factores de vulnerabilidad específicos de la MIRR que están estrechamente ligados a su marginalidad. Es el caso de la atención puesta en la actividad de 
segregación de los residuos, que contribuye fuertemente a la representación negativa de este espacio urbano ( $\mathrm{D}^{\prime}$ Ercole \& Sierra, 2008). A diferencia del proyecto Dipecho, los objetivos expuestos engloban dimensiones que no están directamente ligadas a la existencia de amenazas sísmicas y morfoclimáticas. La noción de ordenamiento territorial integral está más desarrollada en éste, debido sin duda a las orientaciones de los dos actores principales: Cenca, que es una ONG especializada en la planificación urbana y la vivienda, y el IMP, que es un organismo de planificación territorial. El proyecto Dipecho, por su parte, atañe directamente a la preparación de la población ante un eventual evento mayor. No apunta a modificar la actividad de los residentes ni a reorganizar la ocupación del territorio, sino a modificar la percepción del ambiente y el comportamiento de la población.

Por ende, las temporalidades de intervención no responden a las mismas exigencias. El proyecto IDRC intenta modificar los modos de ocupación y el funcionamiento del espacio urbano. Requiere de una duración tanto más larga por cuanto tiene oficialmente previsto organizar una investigación y una elaboración participativa de las soluciones. En los hechos, las que se imponen son la representación oficial del riesgo y las metodologías de países del Norte. Esto se traduce sobre todo a través de la importancia dada a las medidas de prevención del riesgo en términos de planificación y de acondicionamiento urbano a largo plazo. El programa Dipecho, en cambio, tiene una duración reducida: se concentra en los aspectos de preparación frente a los desastres y se adapta a las condiciones locales. Busca reducir el riesgo presente, aceptando la realidad de la ocupación del suelo, sin pretender un ideal territorial.

Por esta razón, el territorio de las operaciones de prevención también difiere: mientras el proyecto Dipecho trabaja con la población que ocupa los terrenos más inestables y más expuestos a las amenazas (sobre todo hundimientos) al borde del Rímac, el IDRC los excluye. En efecto, las autoridades municipales prohíben el fortalecimiento de las edificaciones en las zonas catalogadas como «riesgosas» en los planes de zonificación de la Municipalidad Metropolitana de Lima (ver artículo de J. Robert y A. Sierra, en este volumen, pp. 595-621). Incluso si la municipalidad no ejecuta su planificación, y no reubica a los ribereños, la reducción de los factores físicos de vulnerabilidad previstos dentro del marco del proyecto IDRC (fortalecimiento de las edificaciones) entraría en contradicción con esta planificación municipal. Este proyecto busca entonces mejorar las condiciones de existencia de la población dentro de los límites de y en conformidad con el ideal territorial oficial.

Estos dos proyectos de alcance local apuntan a reducir la vulnerabilidad. A través de este objetivo, a pesar de metodologías radicalmente diferentes, proponen un enfoque todavía poco desarrollado en Lima, que viene a alimentar una gestión del riesgo todavía incompleta. 


\section{DEL CONFLICTO A LA CONCERTACIÓN: HACIA UNA GESTIÓN INTERINSTITUCIONAL DE LOS RIESGOS}

Recientemente han aparecido proyectos de implementación de políticas de ordenamiento territorial y de gestión del ambiente en diferentes escalas. Estos proyectos parecen tomar en cuenta la problemática de gestión de los riesgos de manera más integral.

Hasta ahora, la gestión de los riesgos ha sido considerada a través de políticas sectoriales y de acciones muy localizadas. Sin embargo, algunos mecanismos están naciendo en Lima, que toman en cuenta los riesgos dentro de un tema más amplio: el ambiente urbano. A veces en torno a mecanismos institucionales promovidos por el ministerio del Ambiente, otras veces tras conflictos (como fue el caso del tratamiento de las aguas servidas), nacen colaboraciones entre instituciones. En todos los casos, estas permiten a los diferentes actores urbanos trabajar juntos para mejorar el manejo de la ciudad, su medio ambiente y reducir los riesgos a los cuales están expuestas las poblaciones.

\section{1. Tratamiento de las aguas servidas en Lima: una gestión conflictual del riesgo}

La gestión del agua potable y de las aguas servidas de la capital peruana es efectuada por la empresa estatal Sedapal (Servicio de Agua Potable y Alcantarillado de Lima). Sin embargo, tras un conflicto relativo a las aguas servidas, las municipalidades y los gobiernos regionales participan en la elaboración de esta gestión (Durand, en prensa).

Este conflicto tiene su origen en el hecho de que solamente se trata el $10 \%$ de las aguas servidas de la aglomeración urbana de Lima. Lo que queda es directamente vertido en los ríos, en el mar, o utilizado para irrigar zonas agrícolas. Sedapal tenía proyectado concentrar el $56 \%$ de las aguas servidas colectadas, es decir $10 \mathrm{~m} / \mathrm{s}$, sobre la playa La Taboada, al noroeste del aeropuerto del Callao. El objetivo inicial era construir una planta de tratamiento para tratar los efluentes de los 5 colectores existentes, gracias a la construcción de una nueva canalización: el interceptor norte.

En marzo de 2008, tras la ruptura del colector Costanero en el distrito de San Miguel, el interceptor norte fue puesto en servicio de manera precipitada (fig. 7). Entonces, el 56 \% de las aguas servidas de la ciudad se vertieron en la bahía del Callao sin tratamiento, exponiendo la población ribereña de esta bahía a un gran riesgo sanitario. También se expuso a un riesgo ambiental importante los recursos en agua de la ciudad y el humedal de Ventanilla. Esta zona ya era muy vulnerable por la existencia de numerosos focos de contaminación y de molestias: botaderos, vertimientos de aguas servidas no tratadas, aeropuerto, puerto y autopista. Además, es una zona importante para el abastecimiento alimentario de la ciudad, pues a algunas decenas de metros de los colectores se encuentra el terminal pesquero del Callao y numerosas zonas agrícolas. 


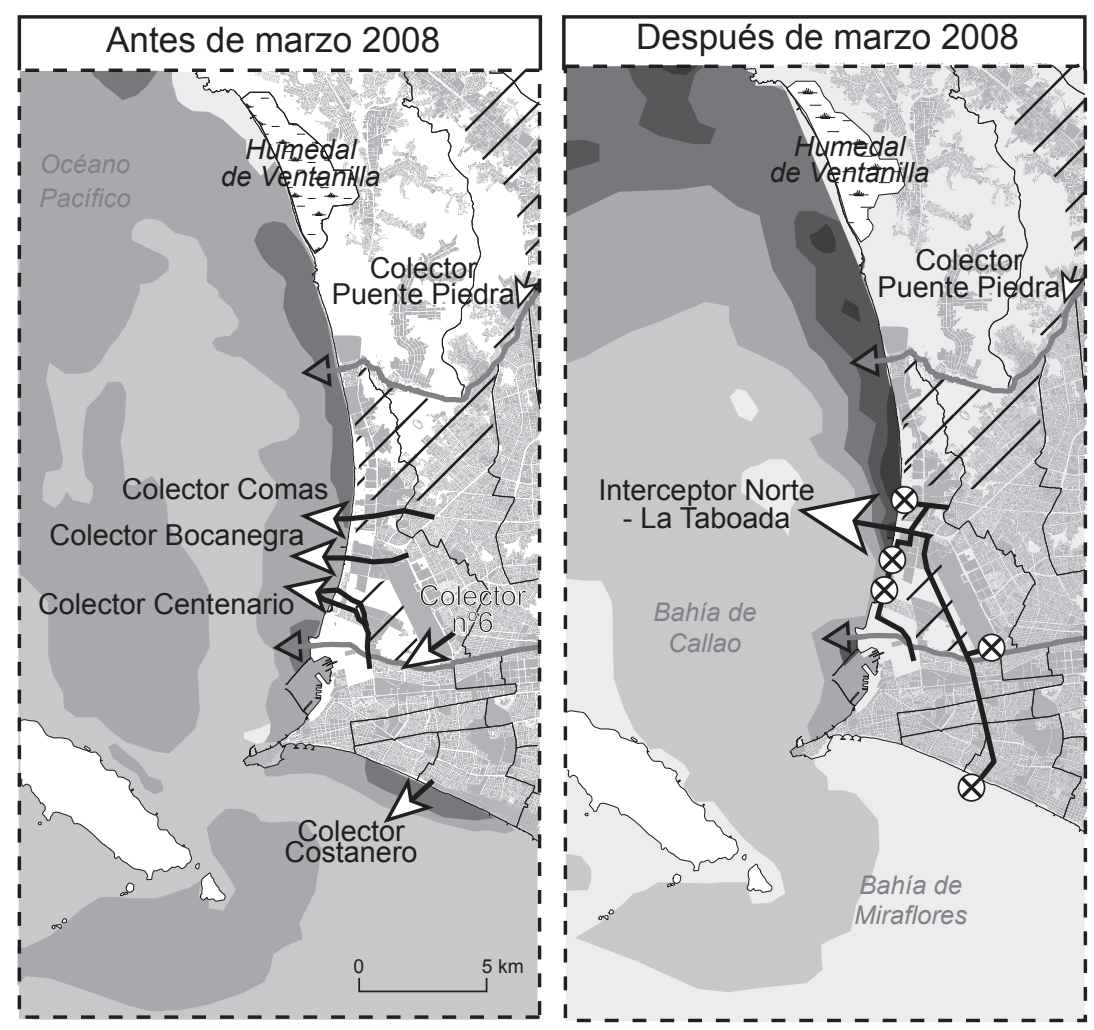

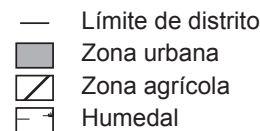

Vertimientos de aguas residuales

$\varangle$ Por canalización de SEDAPAL

$\checkmark$ Por río

Q Vertimiento eliminado

Contaminación marina, sólidos en suspención, en $\mathrm{g} / \mathrm{m} 2$

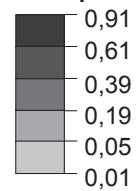

Fuente: INEI, SEDAPAL, IMP, 2005, Ministerio de vivienda, 2006, DIGESA, 2007

Figura 7 - Sistema de evacuación de las aguas servidas de Lima/Callao

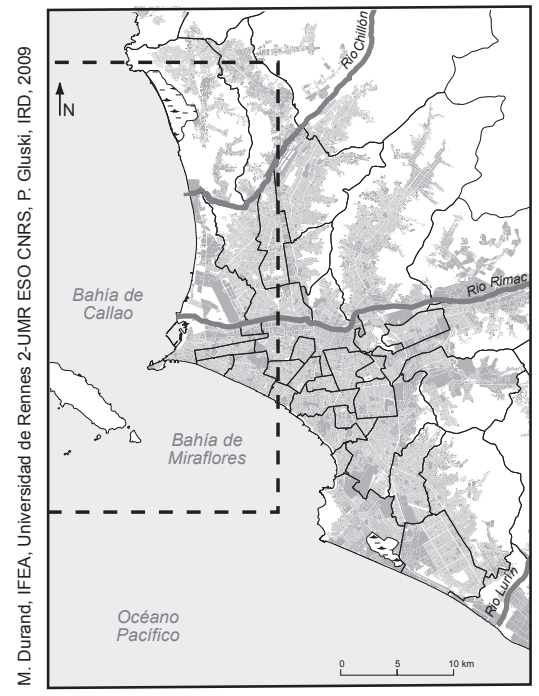


Esta situación de riesgo ambiental y sanitario dio origen a numerosas protestas por parte de los habitantes, mucho más allá de las movilizaciones habituales. Sin embargo, la ausencia de representantes de la población (municipalidades, asociaciones, etc.) en el directorio de Sedapal, hace que las expresiones populares no tengan sino poco impacto sobre las decisiones de la empresa de agua. De este modo, ha sido capital el ingreso de un nuevo actor en el conflicto. Se trata del gobierno regional del Callao, recién creado en 2004, y que está en busca de legitimidad política. Este se lanzó en el conflicto y ha jugado el rol de intermediario entre el Estado peruano y las poblaciones locales. Presionado por los medios de comunicación, el ministerio de Vivienda, Construcción y Saneamiento, del cual depende Sedapal, desbloqueó un presupuesto especial para la construcción de una planta de tratamiento de aguas residuales en dos años. En setiembre de 2008, la empresa española ACS ganó la licitación, proponiendo la solución menos costosa: un simple tratamiento primario y una canalización de $3 \mathrm{~km}$ para evacuar las aguas servidas en alta mar.

A pesar de las protestas del nuevo ministro de Vivienda en julio de 2009 sobre la ineficiencia de tal tratamiento, este no pudo hacer cancelar el contrato firmado por su antecesor. Desde el inicio del proyecto, el único objetivo del Estado peruano fue la descontaminación de la bahía de Miraflores, y poder brindar playas limpias a las poblaciones acomodadas de Lima. Esto se ha hecho en detrimento de la bahía del Callao, donde viven poblaciones más pobres. Sin embargo, la magnitud que tomó la movilización, el apoyo mostrado por las autoridades del Callao, así como la fuerte identidad local, hicieron retroceder al gobierno nacional. De este modo, la movilización de diferentes actores locales ha permitido reducir en parte el riesgo sanitario con la construcción del futuro colector de $3 \mathrm{~km}$ para evacuar las aguas residuales. Sin embargo, no se resuelve todavía el problema del tratamiento de esas aguas.

\section{2. Los organismos de cuenca: el río Chillón}

Además de los conflictos, algunos mecanismos institucionales, voluntaristas, permiten reducir los riesgos ambientales a través de la gestión del agua. En efecto, esta debe tener en cuenta las contaminaciones, las crecidas de ríos, las inundaciones y diversos eventos hidromorfológicos (lavas torrenciales o huaycos, deslizamientos, hundimientos). Como el ciclo del agua no funciona según los límites administrativos, numerosos países han implementado organismos de concertación que agrupan a todos los actores de una cuenca — territorio hidrológico- con el objeto de mejorar la gestión de este recurso y disminuir los riesgos asociados a ella. El Perú reflexiona sobre la creación de instituciones de gestión integral e integrada por cuenca, bajo el impulso de dos organismos de reciente creación: la ANA (Autoridad Nacional del Agua) encargada del manejo de los recursos en agua, y el ministerio del Ambiente.

Existen ya algunas experiencias tal como el GTE (Grupo Técnico Estratégico) del río Chillón, en la parte norte de Lima, implementado con el apoyo logístico y la 
experticia de la ONG peruana Alternativa. El GTE integra las municipalidades, los gobiernos regionales, los agricultores, diversas asociaciones, empresarios y universidades. Sin embargo, no tiene ningún poder de reglamentación y dispone de muy pocos recursos para actuar. Su herramienta principal es la redacción de diagnósticos, la elaboración de planes y la organización de la concertación entre actores para mutualizar los recursos. Su única fuerza es su capacidad de convencimiento.

Por un lado, el GTE de la cuenca del río Chillón ha implementado una comisión de minería que trabajó sobre la reducción de los riesgos (contaminación, deslizamientos, etc.) producidos por la pequeña minería informal presente en su territorio. Otra comisión se ha encargado de redactar un Plan de Ordenamiento Territorial, cuyo objetivo fue orientar los documentos de urbanismo de las municipalidades11 en busca de una gestión integrada del agua, de la protección de recursos naturales y de la disminución de los riesgos ligados al agua.

Por otro lado, el trabajo de coordinación ha permitido organizar operaciones conjuntas de limpieza de las riberas del río Chillón, infestadas de basura y de escombros, para reducir el riesgo sanitario y la posibilidad de construir viviendas de manera informal sobre estos suelos inestables. En este marco, las riberas del río también han sido reforzadas para mitigar el riesgo de inundación (PUCP, 2009).

\section{2. Gestión integral e integrada del ambiente local}

El caso anterior nos enseña los límites de la gestión de un territorio que supera los límites políticos tradicionales. No obstante, el proceso participativo que este promueve es cada vez más aplicado en el ámbito de los territorios municipales tradicionales para hacer frente a riesgos múltiples.

Este modo de gobierno participativo es promovido por el ministerio del Ambiente a través del Sistema Local de Gestión Ambiental (SLGA) implementado a nivel de las municipalidades, como en la de Comas (Lima). Este sistema se articula en torno a una certificación de Gestión Ambiental Local Sostenible (GALS) otorgada por el ministerio, que permite a las municipalidades acceder a financiamientos adicionales. Para obtener esta certificación, las municipalidades tienen que organizar una concertación dentro de una CAM (Comisión Ambiental Municipal), que reúne a todos los actores locales, públicos, privados y asociativos. Esta comisión está encargada de redactar un diagnóstico ambiental y proponer luego políticas ambientales.

La fuerte dependencia del SLGA respecto de las municipalidades es la principal debilidad de esta institución. Su capacidad de acción es limitada por carecer

11 Cuando hablamos de «municipalidades» nos referimos a la vez a las municipalidades distritales y a las provinciales, con mecanismos y herramientas a veces diferentes. En varios casos, la legislación peruana no diferencia estas dos instituciones y las considera globalmente como «gobiernos locales». 
de recursos financieros propios y no tener competencia normativa. Igual que el sector ambiental tradicional, la ejecución de estas políticas pasará siempre a un segundo plano en relación con otras, tales como las políticas urbanas o las políticas económicas.

A pesar de una eficiencia relativa, el SLGA es de todos modos un grupo de presión importante. Al organizar e institucionalizar la concertación y el diálogo, la CAM permite fortalecer la problemática ambiental y busca una reducción de los riesgos de origen natural y antrópico.

\section{CONCLUSIONES}

Lima es una metrópoli dividida, cuya expansión es fuertemente consumidora de espacio, y donde se desarrollan cierto número de acciones dirigidas hacia la lucha contra los riesgos. La disparidad de los recursos de las autoridades municipales, así como de los problemas que hay que manejar, favorecen la fragmentación de tales acciones. Entonces se implementan iniciativas aisladas, en diferentes escalas y con diferentes actores. Generalmente, la población es la promotora de estas iniciativas, reivindicando sus derechos ante las autoridades.

Tanto la puesta en perspectiva de los riesgos a escala de la aglomeración urbana, como la puesta en coherencia de estas acciones de lucha contra los riesgos, se encuentran en sus inicios. Este artículo abre la vía a una reflexión más sistemática sobre los espacios riesgosos y los espacios vulnerables en Lima. Permite mostrar la existencia de cinco relaciones que deberán ser profundizadas:

- La relación entre gestión del riesgo y desafíos del desarrollo urbano: así, la prioridad otorgada al ordenamiento de la quebrada Quirio en el asentamiento humano Nicolás de Piérola (Lurigancho-Chosica), la representación de un riesgo hídrico sobre el Chillón o la planificación preventiva a lo largo del Rímac parecen estar ligadas a la protección del eje de la Carretera Central, al control de la expansión urbana y al desarrollo de un eje entre Lima y Callao. ¿Se puede generalizar esta relación?

- Entre reducción de riesgos y movilización social: las acciones efectuadas dependen, en los casos analizados, de la iniciativa de la población, de responsables locales o de organismos como las ONG y, más bien pocas veces de las autoridades metropolitanas o distritales. ¿Están verdaderamente ausentes estas últimas? ¿Qué influencia tienen sobre los actores locales?

- Entre riesgo e integración de márgenes urbanas: la representación espacial del riesgo así como la espacialización de las acciones efectuadas atañe a las márgenes urbanas en el sentido físico y social (Sierra \& Tadié, 2008). ¿En qué medida las acciones de prevención siguen una lógica de integración a una sociedad normalizada, ideal?

- Entre organización político-administrativa y vulnerabilidades: el espacio limeño está fragmentado y los casos analizados muestran una ausencia de acción de las autoridades municipales, una falta de coordinación, o conflictos de 
competencia. ¿La organización territorial e institucional, así como el proceso de toma de decisiones, terminan haciendo vulnerable al conjunto de la metrópoli o pueden ser fuente de resiliencia a través de la capacidad de auto-organización a escala local?

- Entre la gestión de riesgos y una gestión más global del ambiente: la reciente creación del ministerio del Ambiente (2008) y la afición creciente a nivel mundial por esta temática lleva a los poderes públicos a tratar los riesgos cada vez más a través del filtro de las políticas ambientales. ¿En qué medida este elemento puede permitir el mejoramiento de la gestión de riesgos y su mejor integración con las otras políticas públicas o, al contrario, su dilución dentro de un conjunto demasiado amplio?

Estas experiencias muestran también que las acciones efectuadas hasta ahora se focalizan en espacios donde el riesgo es altamente percibido. Los espacios de intervención son casi exclusivamente aquellos donde la amenaza está muy presente, o incluso donde se han constatado daños recientes. Este enfoque del riesgo, centrado sobre la amenaza (Cartier et al., 2008), favorece la segmentación sectorial y espacial de las acciones de lucha contra el riesgo. Esta segmentación y la ausencia de una política global, no pueden sino contribuir a aumentar la vulnerabilidad de la aglomeración.

\section{Referencias citadas}

CARTIER, S., VINET, F. \& GAILLARD, J-C., 2008 - Introduction, Maître du monde ou maître de soi ? In: Vulnérabilités sociétales, risques et environnement (A. Peltier \& S. Becerra, eds.): 9-20; París: Éditions L'Harmattan.

CONAM, 2008 - Guía para la certificación de municipalidades con gestión ambiental local - certificación GALS, 77 pp.; Lima: CONAM.

COOPI, 2008 - Estudio de identificación de zonas de peligro y de vulnerabilidad en el Cercado de Lima y El Agustino, Provincia de Lima, 83 pp.; Lima: Proyecto Dipecho.

D'ERCOLE, R. \& SIERRA, A., 2008 - Enjeux urbains contradictoires et vulnérabilité accrue dans un espace marginal péricentral : la rive gauche du Rímac à Lima (Pérou). Autrepart, 45: 105-122.

DURAND, M., en prensa - Inégalités écologiques et eaux usées à Lima. In: L'eau mondialisée (G. Schneier-Madanes, ed.); París: La Découverte.

EMILIANOFF, C., 2006 - Connaître ou reconnaître les inégalités environnementales ? Travaux et documents, $\mathbf{n} .^{\circ}$ 25: 35-43; Rennes: UMR ESO CNRS.

INDECI, 2006 - Manual básico para la estimación del riesgo, 85 pp.; Lima, Perú: Instituto Nacional de Defensa Civil (Indeci). Dirección Nacional de Prevención. http://bvpad.indeci.gob.pe/doc/pdf/esp/doc319/doc319.htm

PUCP, 2009 - Conflictos en el uso del espacio, caso del parque lineal Chillón de Comas, 66 pp.; Lima: PUCP, GTE Chillón, Alternativa, IFEA. Rapport des étudiants de Géographie

SIERRA, A. \& TADIÉ, J. (eds.), 2008 - La ville face à ses marges. Autrepart, 45: 220 pp.; París: IRD-Colin, En línea: http://www.autrepart.ird.fr/editos/editos/edito45.htm 